Editorials

\title{
American Journal of Agricultural and Biological Sciences: Ten Years Later
}

\author{
Marco Landi \\ Department of Agriculture, Food and Environment, University of Pisa, Pisa, Italy
}

Ten years after the first issue, the American Journal of Agricultural and Biological Sciences (AJABS) continued to flourish, a clear indication of the validity of Science Publications project. Many scientists have contributed in this first decade to AJABS editorial life and to date more than 500 papers have been published with an encouraging rate of 0.73 cites per doc. Furthermore, the high rate of manuscript rejected testimony the willingness of AJABS to maintain a high level of scientific quality. I have been recently appointed as Associate Editor of AJABS. First of all, I would like to thank the Publications Committee of Science Publications for my selection. I am aware that as Associate Editor I will have key tasks, especially during the temporary vacancy of the Editor-in-Chief. In this framework, I have tried to boost the scientific level of the Editorial Board believing that the scientific appeal of the Editorial Members is the first prerequisite for a highrank scientific journal. Dr. Pellegrini was recently invited to join to our Editorial Board as an expert on global change effects on plant physiology, in particular the effect of increasing ozone concentration on plant; Dr. Calatayud was invited as expert on crop science, grafting and salinity; Dr. Sebastiani has a strong background in molecular biology, functional genomics and transcriptome analyses; Dr. Benelli is an expert in entomology, with special reference to biological control, integrated pest management, mosquito control with nanoformulations and insect pheromones, and Dr. Papadakis as an expert on horticulture, plant nutrition, fruit quality and orchard management techniques. I want also to express my sincere gratitude to the "older" Editorial Members, Dr. Rahman, Sinha, Srivastav, Testa, Xiong and Zamorano for the great work they have done for AJABS till today. However, further challenges still deserve attention. In particular, some research field need to be covered better with other expert on matter. This is of great importance for our journal in order to increase its scientific impact. Overall, I hope all of you want to be part of this exciting project. 